\title{
P-type ATPases use a domain-association mechanism to couple ATP hydrolysis to conformational change
}

\author{
Xuejun C. Zhang ${ }^{1,2 \bowtie}$, Hongwei Zhang ${ }^{1,2}$ \\ 1 National Laboratory of Biomacromolecules, CAS Center for Excellence in Biomacromolecules, Institute of \\ Biophysics, Chinese Academy of Sciences, Beijing 100101, China \\ 2 University of Chinese Academy of Sciences, Beijing 100049, China
}

Received: 7 November 2018 / Accepted: 15 January 2019 / Published online: 29 April 2019

\section{INTRODUCTION}

Since J. Skou discovered the $\mathrm{Na}^{+}-\mathrm{K}^{+}$pump in peripheral nerves more than 60 years ago (Skou 1957), the number of isolated phosphorylation-type ATPases (P-type ATPases or P-ATPases) has grown largely (Palmgren and Nissen 2011). While over 160,000 entries of P-ATPases from both eukaryotes and prokaryotes have currently been documented in the InterPro database, they are especially widespread and rich in subtypes in eukaryotes (Kuhlbrandt 2004). A common function of the members from this family is to convert energy derived from ATP hydrolysis into electrochemical potential by transporting cations across plasma membranes or sequestrating them into membranecompartmented organelles. In turn, generating and maintaining such cross-membrane electrochemical potentials are pivotal to many cellular processes, including operations of secondary active transporters (Zhang et al. 2018a) and the cellular signaling system comprising numerous types of ion channels (Zhang et al. 2018b). In short, P-ATPases are a ubiquitous, highly conserved, and probably ancient family of transporters that includes many members essential to their corresponding organisms.

In the late 1960s and early 1970s, R. Albers and R. Post proposed a basic model describing the functional cycle of P-ATPases (Albers 1967; Post et al. 1972). A signature feature of this so-called Post-Albers cycle is the existence of a phosphoenzyme intermediate containing a phosphorylated Asp residue. In agreement

$\bowtie$ Correspondence: zhangc@ibp.ac.cn (X. C. Zhang), with the more general "alternating access" model for all transporters (Jardetzky 1966), the Post-Albers model proposes that a P-ATPase assumes both a cytosol-facing $\mathrm{E}_{1}$ conformation with high affinity to the substrate cations, as well as an outward-facing $\mathrm{E}_{2}$ conformation with low affinity to its substrates (Fig. 1A; here, "outward" stands for the extracellular or lumenal direction). The core concept of this model has been supported by numerous biochemical and biophysical studies, remaining valid to this day. Among the supportive evidence is a large volume of data from structural biology studies.

Beginning from the turn of the new millennium, structural biology has generated a series of highresolution snapshots covering nearly the entire PostAlbers cycle (Bublitz et al. 2011; Toyoshima 2008; Toyoshima and Cornelius 2013; Toyoshima et al. 2000; Winther et al. 2013). Recently, using an X-ray solvent contrast modulation technique, Toyoshima and coworkers refined the overall picture of the conformational changes occurring within the $\mathrm{Ca}^{2+}$-ATPase 1 of skeletal muscle sarco(endo)plasmic reticulum (SERCA1), which had been the model system for understanding the functional cycles of P-ATPases (Norimatsu et al. 2017). This updated model shows how important the interplay between the P-ATPase and its surrounding lipid bilayer is, even though certain forms of crystallization artifacts can still not be ruled out absolutely. A recent study on the $\mathrm{Ca}^{2+}$-ATPase from Listeria monocytogenes (LMCA1), using the singlemolecule fluorescence resonance energy transfer (smFRET) technique, describes in detail the dynamics of the conformational changes of the P-ATPase, in addition 
A

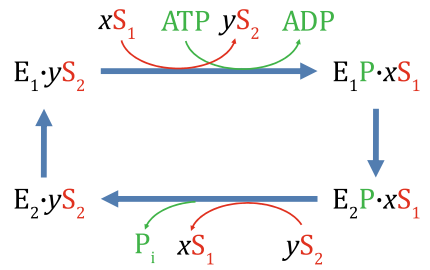

$\mathrm{S}_{1}, \mathrm{~S}_{2}:$ mono cations $x>y$

B

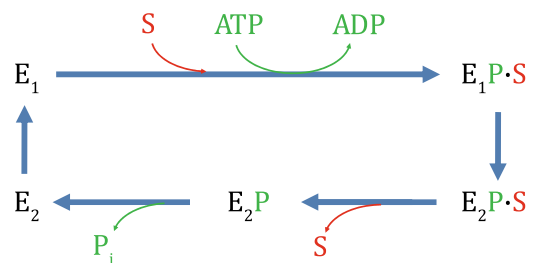

Fig. 1 Post-Albers cycle of the P-ATPase. A The classical PostAlbers cycle. $E_{1}$ and $E_{2}$ represent conformations corresponding to the kinase and phosphatase states, respectively. $S_{1}$ and $S_{2}$ represent the mono-cationic substrates and counter ions, respectively. B A simplified version of the Post-Albers cycle. Exchange of substrate cation and counter ions is represented by the loading and releasing of a single effective substrate (S)

to consolidating data from previous biochemical studies (Dyla et al. 2017). However, most of previous information on the structures and dynamics of P-ATPases was obtained in the absence of a bona fide cellular membrane, whose presence is critical for imposing both electrochemical potential and lateral tension among other environmental factors to the target membrane proteins. Such experimental manipulation may cause subtle yet significant changes to the structures and properties of the proteins (Liu et al. 2018). A fundamental question about P-ATPases that remains debated is as to how the ATP hydrolysis and substrate transport are energetically coupled. Here, we propose a thermodynamic model to address this question in light of currently available structural and dynamics information.

\section{OVERALL STRUCTURE OF P-ATPASE}

A typical P-ATPase contains three cytosolic domains and one transmembrane domain (Fig. 2A) (Palmgren and Nissen 2011; Toyoshima et al. 2000). The cytosolic domains are nucleotide-binding $(\mathrm{N})$ domain, phosphorylation (P) domain, and actuator (A) domain. The transmembrane (M) domain typically contains 10 transmembrane (TM) helices (M1-M10). Of these helices, M1-M6 are conserved among members of the P-ATPase family. In contrast, helices M7-M10 mainly function as a membrane anchor to provide stability to the M-domain (Norimatsu et al. 2017); they may also contribute to substrate specificity (Palmgren and Nissen
2011), thus varying from protein to protein. In certain P-ATPases, M7-M10 assuming the anchoring role are replaced by $\mathrm{N}$-terminal TM helices (Gourdon et al. 2011) or supplemented with additional helices (Bublitz et al. 2011). Importantly, the M4 helix breaks at the middle (e.g., in a PEGL motif conserved in $\mathrm{Ca}^{2+}$. ATPases), resulting in two segments namely extracellular/lumenal M4a and cytosolic M4b. Similarly, a bulge is observed in the middle of the helix M6. These two irregularities in TM helices contribute to the formation of a membrane-embedded binding pocket for the substrate cations. To be analogous to descriptions of other types of transporters, it may be helpful to divide the $\mathrm{M}$-domain into $\mathrm{N}$ - and C-terminal TM subdomains, $\mathrm{N}_{\mathrm{TM}}$ (formed by M1-M4a) and $\mathrm{C}_{\mathrm{TM}}$ (M4b-M10), respectively. The substrate-binding site is located between $\mathrm{N}_{\mathrm{TM}}$ and $\mathrm{C}_{\mathrm{TM}}$, albeit being associated more with $\mathrm{C}_{\mathrm{TM}}$. The conformational changes at the $\mathrm{N}_{\mathrm{TM}}-\mathrm{C}_{\mathrm{TM}}$ interface during the $E_{1}-E_{2}$ cycle permits alternating access of this binding site to either the cytosol or extracellular/ lumenal space.

The cytosolic A-domain represents an insertion to the $\mathrm{N}_{\mathrm{TM}}$, connected to helices M2 and M3 (in some subtypes also to M1) through flexible linkers, which constrain the maximum distance between the A- and M-domains. Similarly, the P-domain represents an insertion to the $\mathrm{C}_{\mathrm{TM}}$. In turn, the cytosolic $\mathrm{N}$-domain comprises an insertion to the P-domain through a strongly conserved hinge of two antiparallel peptide strands, thus pointing away from the membrane. Importantly, the TM helices $\mathrm{M} 4 \mathrm{~b}$ and M5 of $\mathrm{C}_{\mathrm{TM}}$ extend into the cytosol and constitute a portion of the P-domain (Palmgren and Nissen 2011). Thus, both $\mathrm{C}_{\mathrm{TM}}$ and $\mathrm{P}$-domain appear to move together as a semi-rigid body.

According to the refined picture proposed by Toyoshima and co-workers (Norimatsu et al. 2017), it is the $\mathrm{C}_{\mathrm{TM}}-\mathrm{P}$ rigid body that rock-and-rolls relatively to the A-domain as well as to the membrane bilayer, thus generating alternating pathways for the substrate cations. This work clearly illustrates the importance of the choice of viewpoint applied during structural comparisons. Typically, when analyzing the conformational changes occurring within a (soluble) protein, researchers superimpose the largest rigid part of the protein and look for conformational differences occurring in other smaller regions. However, for an integral membrane protein, it may not be correct to assume that the largest rigid piece of a membrane protein always remains static in the lipid bilayer. Instead, the reference point should be the overall membrane bilayer plus protein components that are more-or-less static within the membrane regardless of their sizes. In the case of the P-ATPase, the $\mathrm{C}_{\mathrm{TM}}-\mathrm{P}$ rigid body functions as a rocking player switching 
A

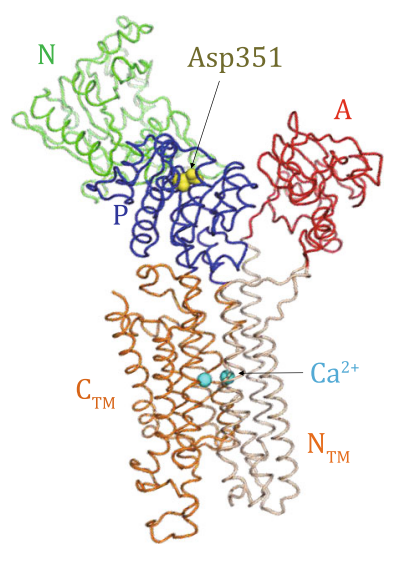

SERCA1(1SU4)
B

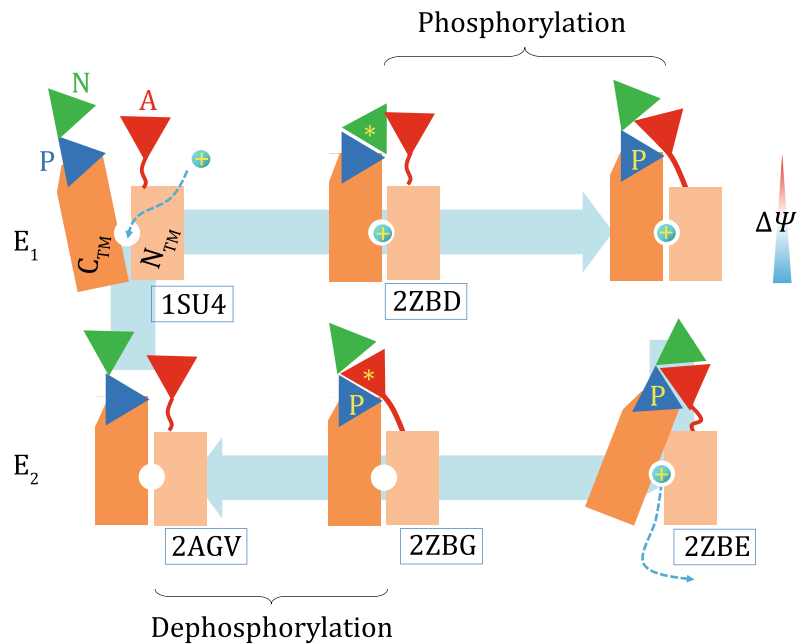

Fig. 2 Cycle of conformational changes of the P-ATPase. A Representative structure of SERCA1 (PDB ID: 1SU4). B Cartoon diagram of the cycle of conformational changes of the P-ATPase. The effective substrate cation is indicated as a cyan sphere. The cytosolic N-, P-, and A-domains are represented as triangles and colored in green, blue, and red, respectively. Transition states of phosphorylation and dephosphorylation are marked with yellow asterisks, and phosphorylated P-domain is marked with a letter "P". PDB IDs of representative crystal structures are included as references

between its $E_{1}$ and $E_{2}$ states. This movement appears fairly large, being amplified by the cytosol-protruding Pand $\mathrm{N}$-domains. In contrast, the overall structure of the $\mathrm{N}_{\mathrm{TM}}$ appears to be less dynamic relative to the membrane, especially in the direction of the membrane normal. During such a conformational change, the $\mathrm{C}_{\mathrm{TM}}$ helices change their tilting angles in the membrane, and the P-domain changes its position and orientation relatively to the A-domain (Fig. 2B). In addition, the structurally observed protein-phospholipid interactions are governed primarily by a belt of Trp as well as basic residues. Interestingly, the interaction between the Trp residues and surrounding lipids are not mediated by hydrogen bonds with the polar head groups (Norimatsu et al. 2017), in contrast to what is often assumed. It is likely that Trp residues exert their stabilizing effect on membrane proteins through interactions between the intrinsic (and induced) electric dipoles of their indole sidechains and the strong local electric field from the head-group phosphates of the surrounding lipid molecules (Zhang and Li 2019).

Interactions between the three cytosolic domains change in response to the substrate loading and releasing. The "key" Asp residue in a conserved DKTGT motif of the P-domain (e.g., Asp351 in SERCA1) alternates between phosphorylated and dephosphorylated states. On the one hand, the $\mathrm{N}$-domain functions as a built-in kinase (enzyme-1 or $E_{1}$ ) to transfer the $\gamma$ phosphate group from ATP to the P-domain. This reaction is catalyzed by using an $\mathrm{S}_{\mathrm{N}} 2$ mechanism mediated by two $\mathrm{Mg}^{2+}$ co-factors bound to the P-domain (site I, stabilizing the $\gamma$-phosphate as well as the key Asp residue) and N-domain (site II, binding with the $\alpha$ - and $\beta$-phosphates of either ATP or ADP) (Sorensen et al. 2004). On the other hand, by using its conserved TGES motif, the A-domain functions as a cis-phosphatase (enzyme-2 or $\mathrm{E}_{2}$ ) to catalyze dephosphorylation. This latter reaction also utilizes an $\mathrm{S}_{\mathrm{N}} 2$ mechanism but is facilitated by only one $\mathrm{Mg}^{2+}$ co-factor (bound to site I) (Toyoshima et al. 2007). Since both the kinase and phosphatase modules target the same Asp residue in the P-domain, $\mathrm{N}$ - and A-domains have to alternate their intimacy with the P-domain. After each reaction, the corresponding module actively switches with the other module, concerting with the next triggering event (i.e., substrate loading or releasing) to sustain the ATP hydrolysis cycles.

\section{Amphipathic helices}

In a number of P-ATPase structures, an amphipathic helix is clearly observed at the N-terminus of M1, for examples $\mathrm{M}^{\prime}$ ' in both $\mathrm{Ca}^{2+}$-ATPase/1WPG (Toyoshima et al. 2004) and $\mathrm{Na}^{+}-\mathrm{K}^{+}$pump/4HYT (Laursen et al. 2013); and MB' in both ZntA/4UMW (Wang et al. 2017) and CopA/3RFU (Gourdon et al. 2011). These amphipathic helices are likely to anchor the $\mathrm{N}_{\mathrm{TM}}$ subdomain to the lipid bilayer, particularly restricting movement of the N-terminus of M1 to the surface of the cytosolic leaflet. In certain P-ATPases, an additional short amphipathic helix exists at the lumenal end of M9 (Toyoshima et al. 2004). Similar amphipathic helices have been found in several major families of membrane proteins, including secondary active transporters, 
ATP-binding cassette (ABC) exporters (Zhang et al. 2018a), G-protein coupled receptors (GPCRs) (Zhang et al. 2014), and voltage-gated ion channels (VGICs) (Zhang et al. 2018b). Similar to the Trp and basic residues near the membrane surface, these amphipathic helices are likely to play important roles in restricting the mode of conformational changes of their connected TM-helix termini to sliding-only on the membrane surface (Zhang and Li 2019).

\section{SUBSTRATE BINDING}

In a functional cycle of the P-ATPase, substrate binding and releasing are believed to be necessary conditions for the phosphorylation and dephosphorylation events, respectively. However, the two types of events occur at $\sim 45$ - $\AA$ distance from each other (Fig. 2A) (Toyoshima et al. 2000). Thus, a central question in the P-ATPase research field concerns communication mechanism(s) between the substrate-binding and phosphorylation sites (Das et al. 2017).

Structural biology studies have provided essential information to address this question. For instance, SERCA1 contains two $\mathrm{Ca}^{2+}$ binding sites (Toyoshima et al. 2000). Whereas the site- 1 consists of residues from the $\mathrm{C}_{\mathrm{TM}}$, the site- 2 is formed between $\mathrm{N}_{\mathrm{TM}}$ and $\mathrm{C}_{\mathrm{TM}}$. In the substrate-loading step of SERCA1, two $\mathrm{Ca}^{2+}$ ions sequentially replace the bound protons at the binding sites- 1 and -2 . In particular, $\mathrm{Ca}^{2+}$-binding at the site- 2 triggers the phosphorylation step (Jensen et al. 2006). $\mathrm{Ca}^{2+}$ loading is companied by an inward translation (towards the cytosol) and $\sim 20^{\circ}$ inclination of M4b relative to the rest protein structure. The relative movement between M4b and M5 critically affects for the conformation of the P-domain, consequently changing the inter-domain organization and permitting the formation of the catalytic site of the phosphorylation reaction (Jensen et al. 2006).

Commonly, a P-ATPase pumps a net positive charge(s) out of the cytosol. For example, SERCA1a expels two $\mathrm{Ca}^{2+}$ cations in exchange for an influx of 2-3 $\mathrm{H}^{+}$(Obara et al. 2005), with a net output of +1 or +2 electron-units. In the $\mathrm{Na}^{+}-\mathrm{K}^{+}$pump, three $\mathrm{Na}^{+}$ions exchange for two $\mathrm{K}^{+}$, with a net output of +1 (Apell et al. 2011). The expelled cations are often referred to as substrates of the pump, and the cations moving in the opposite direction are considered counter ions. Interestingly, the two $\mathrm{K}^{+}$counter ions of the $\mathrm{Na}^{+}-\mathrm{K}^{+}$pump can be experimentally replaced by other mono-cations, including some organic cations and even two $\mathrm{Na}^{+}$ions, maintaining in an efflux of +1 charge per cycle (Ratheal et al. 2010). Moreover, under physiological conditions, negatively charged lipid molecules, phosphatidylserine (PS), are retained in the inner leaflet of the plasma membrane. In the presence of a negative-inside membrane potential $(\Delta \Psi)$, this orientation of PS represents a high-energy state, and needs to be actively maintained by a special subgroup of P-ATPases called flippases. Exposure of the PS molecules on the extracellular surface of a eukaryotic cell reflects diminished intracellular [ATP] and often serves as an apoptosis signal to be recognized by the immune system. Interestingly, transport of PS by the flippase is equivalent to a net output of a +1 charge (Palmgren and Nissen 2011). Therefore, expelling positive charges appears to be a common, though not absolute, function of P-ATPases (a rare but well-known exception is the $\mathrm{H}^{+}-\mathrm{K}^{+}$pump whose transport process is thought to be electroneutral (Palmgren and Nissen 2011)). Part of the reason of using P-ATPase to expel cations is that this type of functions cannot be performed by the structurally simpler, secondary active transporters both effectively and energetically efficiently.

Substrate cations and counter ions are likely to compete with each other for the same binding site(s) (Apell et al. 2011). Thus, loading of substrate cations in the $E_{1}$ state is coupled with the releasing of the counter ions, and a reverse situation occurs in the $E_{2}$ state. The number of potential binding sites and their affinities to the substrate cations and counter ions depend on the local conformation of the binding pocket for a given state (i.e., $\mathrm{E}_{1}$ or $\mathrm{E}_{2}$ ). For instance, in the structure of SERCA1/1SU4 (in the $\mathrm{E}_{1} \cdot 2 \mathrm{Ca}^{2+}$ state), the two substrate $\mathrm{Ca}^{2+}$ ions are coordinated by four acidic residues as well as other polar groups. Upon releasing of the two $\mathrm{Ca}^{2+}$ cations, all of these acidic residues would possess high $\mathrm{p} K_{\mathrm{a}}$, thus potentially allowing protonation. However, once some of those residues of higher $\mathrm{p} K_{\mathrm{a}}$ get protonated (or bind to $\mathrm{H}_{3} \mathrm{O}^{+}$) first, the $\mathrm{p} K_{\mathrm{a}}$ values of the rest titratable groups decrease because of the positive charges which have been introduced. Consequently, not all of the four acidic residues may get protonated, and less positive charges from the counter ions than that of the substrate ions will be imported into the cytosol. It is reasonable to speculate that the structural and/or electrostatic effect of \pm 1 unit of electric charges in the substrate-binding pocket, which is located in the $\mathrm{N}_{\mathrm{TM}}-\mathrm{C}_{\mathrm{TM}}$ interface, can propagate to the interfaces between the cytosolic domains (Zhang and $\mathrm{Li}$ 2019). Therefore, in the following discussion, we simplify all movement of substrate and counter ions as an efflux of one effective cation (Fig. 1B). Loading and releasing of this effective cation are described as the triggering events for phosphorylation and dephosphorylation, respectively. 
The phosphorylation and dephosphorylation reactions can be considered as the two key events to drive the conformational cycle of the P-ATPase. Conceptually, they resemble the protonation and deprotonation events in proton motive force (PMF)-driven secondary transporters (Zhang et al. 2018a). In other words, the P-ATPase would keep cycling along the path of $\mathrm{E}_{1} \rightarrow \mathrm{E}_{1} \mathrm{P} \rightarrow \mathrm{E}_{2} \mathrm{P} \rightarrow \mathrm{E}_{2} \rightarrow \mathrm{E}_{1}$ regardless of whether substrate binding has occurred, as long as ATP hydrolysis can be uncoupled from substrate transport. In fact, some physiological regulators of P-ATPases are indeed able to induce such decoupling (Bal et al. 2012). Under "normal" physiological conditions, however, the substrate-loading step is mostly likely to be tightly coupled with phosphorylation of the P-domain in order to prevent a futile ATP hydrolysis cycle in the P-ATPase; so is the releasing step to dephosphorylation. For instance, both substrate loading to the M-domain and ATP binding to the $\mathrm{N}$-domain are required for triggering the phosphorylation reaction (Dyla et al. 2017), whereas the order of the two steps is found not to be critical for the reaction (Bublitz et al. 2011). In fact, the cellular concentration of ATP is assumed to be within the millimolar range. It is high enough for the free ATP to replace bound ADP in the $\mathrm{N}$-domain in most of the P-ATPase intermediate states as long as the $\mathrm{N}$ - and P-domains have been dissociated from each other. Furthermore, bound ATP in this modulatory mode has been found to play stimulating roles in most of the functional cycle (Jensen et al. 2006). Similarly, the substrate release was shown to be a pre-condition for dephosphorylation (Dyla et al. 2017).

\section{Differential binding energy $\left(\Delta G_{\mathrm{D}}\right)$}

A characteristic feature of P-ATPases is their ability to transport the substrate cations against a strong electrochemical potential, including a concentration gradient (e.g., as high as $10^{4}$-fold in SERCA1) and/or electrostatic membrane potential (e.g., $\sim 100 \mathrm{mV}$ in $\mathrm{Na}^{+}-\mathrm{K}^{+}$ATPase). Moving of the substrate against its gradient requires both strong affinity (low $K_{\mathrm{d}, \mathrm{L}}$ ) on the loading side of the membrane and weak affinity (larger $K_{\mathrm{d}, \mathrm{R}}$ ) on the releasing side (where subscripts $\mathrm{L}$ and $\mathrm{R}$ stand for loading and releasing, respectively). Such a difference in $K_{\mathrm{d}}$ between the $\mathrm{E}_{1}$ and $\mathrm{E}_{2}$ states of the transporter is necessarily associated with an energy term called differential binding energy $\left(\Delta G_{\mathrm{D}}\right)$ (Zhang et al. 2015),

$\Delta G_{\mathrm{D}} \equiv R T \cdot \ln \left(K_{\mathrm{d}, \mathrm{R}} / K_{\mathrm{d}, \mathrm{L}}\right)>0$.

Here, we follow the convention that a positive energy term corresponds to an endergonic process or step. $\Delta G_{\mathrm{D}}$ is a characteristic parameter of the transporter for a given substrate. On the one hand, in order for the transport to proceed against the uphill energy change, the positive $\Delta G_{\mathrm{D}}$ must be compensated by external energy, e.g., from the exergonic ATP hydrolysis in the case of P-ATPases. Such a statement is arguably a synonym for the energy-coupling mechanism in thermodynamics terms. On the other hand, $\Delta G_{\mathrm{D}}$ constitutes a portion of the change of the chemical potential of the substrate, $\Delta \mu(\mathrm{S})$, during the transport:

$\Delta \mu(\mathrm{S}) \equiv R T \cdot \ln \left([\mathrm{S}]_{\mathrm{R}} /[\mathrm{S}]_{\mathrm{L}}\right)=\Delta G_{\mathrm{L}}+\Delta G_{\mathrm{D}}+\Delta G_{\mathrm{R}}$,

where

$\Delta G_{\mathrm{L}} \equiv-R T \cdot \ln \left([\mathrm{S}]_{\mathrm{L}} / K_{\mathrm{d}, \mathrm{L}}\right)$ and

$\Delta G_{\mathrm{R}} \equiv R T \cdot \ln \left([\mathrm{S}]_{\mathrm{R}} / K_{\mathrm{d}, \mathrm{R}}\right)$.

Through the Gibbs-energy terms of the loading step $\left(\Delta G_{\mathrm{L}}\right)$ and of the releasing step $\left(\Delta G_{\mathrm{R}}\right)$, the substrate concentrations on both sides of the membrane influence the overall rate of the transport process. For instance, a biochemist may optimize the in vitro experimental conditions so that the substrate concentration will be sufficiently high to encourage abundant substrate binding in the $\mathrm{E}_{1}$ conformation while allowing releasing from the $E_{2}$ conformation, thus permitting continuous turnover of the ATP hydrolysis. Nevertheless, physiological conditions are often far away from such an optimized in vitro condition. Importantly, the positive $\Delta G_{\mathrm{D}}$ is associated with conformational changes around the substrate-binding site, resulting in, for example in SERCA1, closing of the M1 "sliding door" (Winther et al. 2013) and loss of the coordination of the substrate cations (Toyoshima et al. 2004) in the $\mathrm{E}_{2}$ conformation.

\section{Free energy landscape plot}

To facilitate discussion on possible energy-coupling mechanisms in thermodynamics terms, we introduce a Gibbs-energy landscape plot of the P-ATPase. As shown in Fig. 3A, two terms are primary: (1) the external energy input is that from ATP hydrolysis, $\Delta \mu$ (ATP); and (2) the total energy gain of all substrates is the summation of $\Sigma_{i}\left(\Delta \mu\left(\mathrm{S}_{i}\right) \pm z_{i} F \Delta \Psi\right)$ over all ions moving across the membrane (where $z_{i}$ is the charge of the $i$ th substrate, and a negative sign is used for counter ions). In our simplified model, only one type of effective substrates is considered. Ratio of the two primary terms represents the efficiency of the energy conversion by the P-ATPase; and the difference between them is defined as $Q_{X}$ ( $\geq 0$, where the subscript $X$ stands for the so-called thermodynamic force in chemical kinetics (Hill 1989)). While $Q_{X}$ represents the heat dissipated by the 
P-ATPase, it should not be mistaken as the experimentally measurable heat during a transport process (which may further include heat release/absorption by the substrates). On the one hand, if releasing of the driving energy is coupled properly with the progress of the functional cycle, $Q_{X}$ is positively correlated with the overall rate of the transport. On the other hand, $Q_{X}$ is negatively related to the efficiency of energy conversion. Consequently, the overall rate is negatively related to the efficiency.

\section{ENERGY OF ATP HYDROLYSIS}

ATP hydrolysis provides P-ATPases with energy of $\sim 50 \mathrm{~kJ} / \mathrm{mol}(\approx 20 R T)$ sufficient to drive substrate transport. As an example of Gibbs-energy changes in one transport cycle, we will first discuss SERCA1, a $\mathrm{Ca}^{2+}$-ATPase that transports two $\mathrm{Ca}^{2+}$ ions per cycle. At the beginning of a transport process (assuming $\Delta \mu(\mathrm{S}) \approx 0$ and $\Delta \Psi \approx 0$ ), the substrate cation does not have to move against a strong concentration gradient, resulting in fast transport (i.e., $Q_{X} \approx \Delta \mu$ (ATP)). As the process proceeds, however, a substrate gradient is gradually built up, and the transport slows down. By the end of this process, the gradient of [S] approaches a $10^{4}$ fold, equivalent to $\Delta \mu(\mathrm{S}) \approx 18 R T$ (i.e., $4 \times 2.3 R T$ multiplying by a factor 2 accounting for the two $\mathrm{Ca}^{2+}$ ions). In addition, as positive charges move across the sarcoplasmic reticulum (SR) membrane, a positive-inlumen electrostatic potential is likely to be built up across the membrane. This $\Delta \Psi$ further slows down the transport, as four positive charges have to move against this potential in each transport cycle (with an uphill energy term of $4 F \Delta \Psi$ ). To maintain high efficiency of $\mathrm{Ca}^{2+}$ sequestration while reducing the cost of electrostatic energy, SERCA1 uses protons as counter ions. Thus, the charge built up across the SR membrane by moving of $\mathrm{Ca}^{2+}$ will be partially compensated by $\mathrm{H}^{+}$. Interestingly, the SR membrane is permeable to protons (Meissner and Young 1980). If we assume that protons (but not other ions) can reach thermo-equilibrium across the SR membrane much more quickly than the transport cycle, the Nernst voltage of protons (i.e., $2.3 R T \Delta \mathrm{pH} / F$ ) equals $\Delta \Psi$. In this case, transporting of the $\mathrm{H}^{+}$counter ions does not cost energy. Consequently, the permeability of $\mathrm{H}^{+}$permits more energy derived from ATP hydrolysis to be used for $\mathrm{Ca}^{2+}$ transport. As we see here, SERCA1 provides a clear example in which counter ions may not affect significantly to the overall gain of electrochemical potential; instead, they are more likely to play a structural role to (at least partially) compensate the imbalance caused by the huge electrostatic forces exerted on the binding-pocket structure upon release of the substrate cations (Toyoshima 2008). Taken together, the maximum energy gain by substrate $\mathrm{Ca}^{2+}$ per functional cycle of SERCA1 is $\sim 18 R T \cdot(1+1 / 2 \Delta \mathrm{pH})$, depending on $\Delta \mathrm{pH}$ across the SR membrane. Although partition of the energy gain of substrates between chemical and electrostatic potentials may differ from one type of P-ATPase to another, similar analyses are likely to be applicable.

Next, for simplicity in thermodynamic analysis, we group all steps associated with the ATP-hydrolysis energy into three steps, namely ATP binding (probably including dissociation of ADP), phosphorylation, and dephosphorylation (including hydrolysis of the aspartylphosphate and dissociation of $\mathrm{P}_{\mathrm{i}}$ ). The corresponding Gibbs-energy terms are $\Delta G_{\mathrm{L}}(\mathrm{ATP}), \Delta G_{\mathrm{ph}}$, and $\Delta G_{\mathrm{deph}}$, respectively, and the summation of the three energy terms equals to the total hydrolysis energy of ATP in cytosol, $\Delta \mu$ (ATP). In the recently reported smFRET experiment on LMCA1 (Dyla et al. 2017), the dephosphorylation step was shown to be irreversible; in contrast, if this dephosphorylation step was blocked experimentally, the phosphorylation step appeared to be reversible. Therefore, in agreement with the fact that normal ATP hydrolysis releases its energy mainly during the cleavage between the $\beta$ - and $\gamma$-phosphate groups, it is reasonable to hypothesize that the major part of the ATP-hydrolysis energy is released in the dephosphorylation step of the aspartyl-phosphate. Moreover, in the absence of electrochemical potential of the substrate (e.g., in an in vitro non-vesicle assay), the phosphorylation step was the rate-limiting step, indicating that the transition from $E_{1}$ ATP.S to $E_{1}$ P.S state is unlikely to represent a dominant step of energy releasing. In contrast, dephosphorylation appeared to be a fast step triggered by the release of the substrate $\mathrm{Ca}^{2+}$ (Dyla et al. 2017). However, as $\left[\mathrm{Ca}^{2+}\right]_{\mathrm{R}}$ increases in a real SR (i.e., $\Delta G_{\mathrm{R}}(\mathrm{S})$ becomes an increasingly positive term), the substrate release will become slower and slower, eventually becoming the rate-limiting step of the functional cycle. In short, the currently available kinetic data suggest a model in which

$\left|\Delta G_{\text {deph. }}\right| \gg\left|\Delta G_{\mathrm{L}}(\mathrm{ATP})+\Delta G_{\mathrm{ph}}\right|$.

One question arising from such an energy conversion scheme is how the released energy from dephosphorylation is coupled to conformational change between $\mathrm{N}_{\mathrm{TM}}$ and $\mathrm{C}_{\mathrm{TM}}$. We hypothesize that the released energy from dephosphorylation is used to dissociate the P-domain from the A-domain $\left(\Delta G_{\mathrm{P}-\mathrm{A}}\right.$ in Fig. $\left.3 \mathrm{~A}\right)$, thus generating a high-energy state between the cytosolic domains. What the positive $\Delta G_{\mathrm{P}-\mathrm{A}}$ means to the binding between the 
A

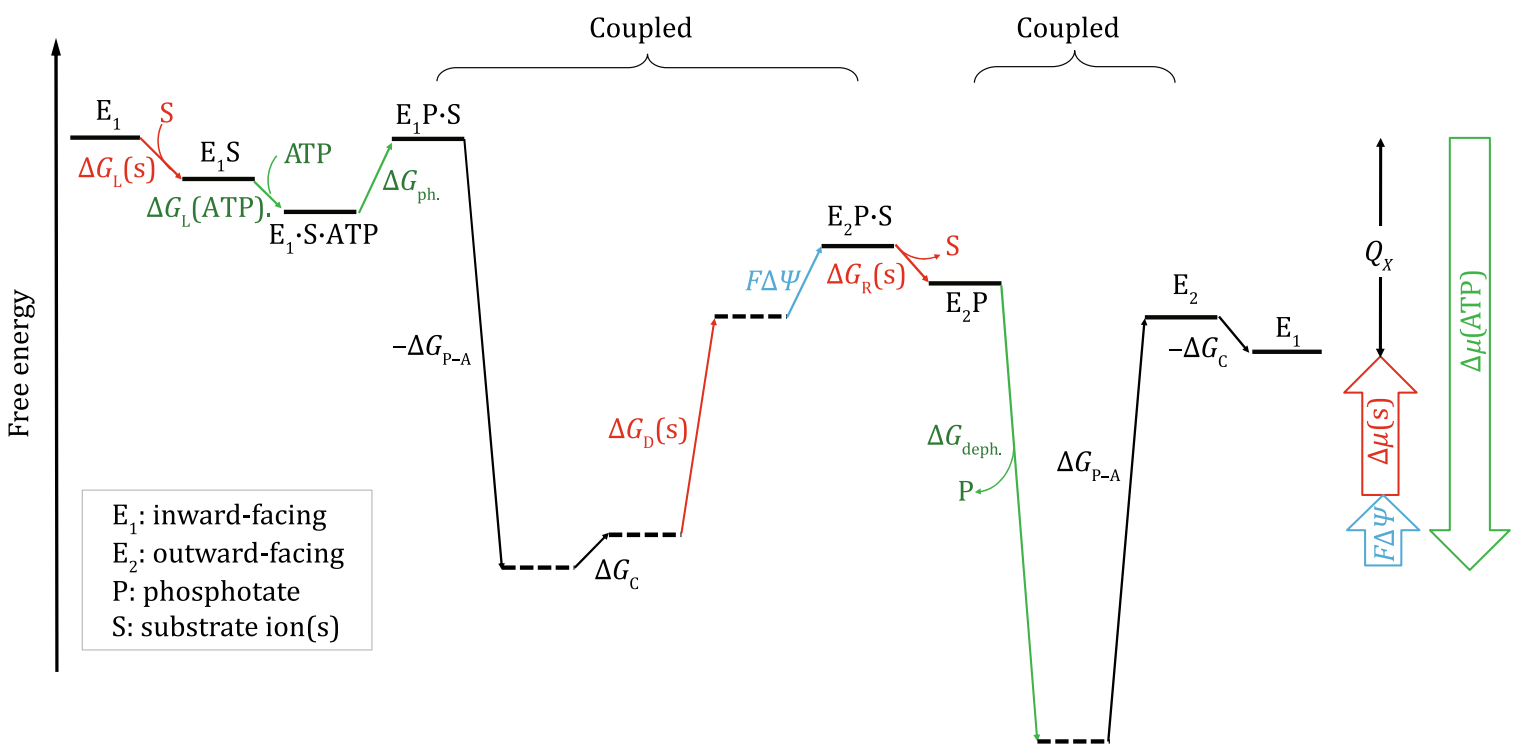

B

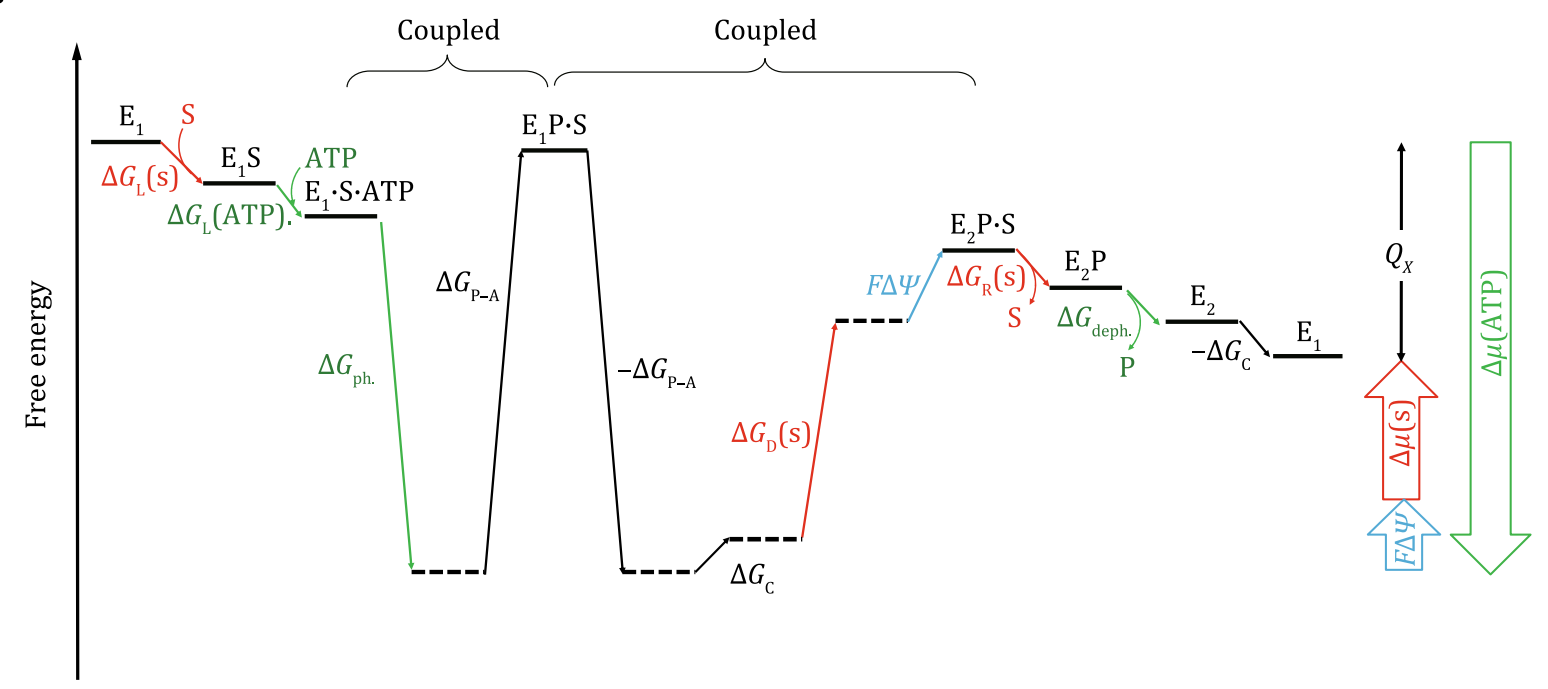

Fig. 3 Energy storage in dissociation. A The energy landscape of the mechanism of "energy storage in dissociation". The main feature of this energy landscape of the P-ATPase can be described as follows: the ATP-hydrolysis energy released during the dephosphorylation step $\left(\Delta G_{\text {deph. }}\right)$ is coupled to the dissociation of the P- and A-domains which is hypothesized to be a major form of energy storage to drive the Post-Albers cycle. B The energy landscape of the "conventional" energy-coupling mechanism. A Gibbs-energy landscape plot describes the thermodynamic relationship between different states. Horizontal lines represent states. Tilted thin arrows represent transitions between states. Green arrows are associated with the chemical potential of ATP. Red and cyan arrows are associated with the chemical and electrostatic potentials of the effective substrate, respectively. Subscripts L and R stand for energy terms associated with loading and releasing, respectively. Collectively, the steps shown in this plot must meet the requirements of the First and Second Laws of thermodynamics. The starting and ending states are identical (i.e., $\mathrm{E}_{1}$ ), only being differed by the energy dissipation (denoted as $Q_{X}$ ) of the P-ATPase transporter during one functional cycle. Notes: (1) Many energy terms in the plot are variable, depending on the cellular/experimental conditions. For example, in case that the chemical potential of the substrate, $\Delta \mu(\mathrm{S})$, becomes very large $\left(i . e .,[S]_{\mathrm{R}} /\right.$ $[\mathrm{S}]_{\mathrm{L}} \rightarrow \infty$ ), the substrate loading/releasing may become exceedingly slow (even reversed). (2) Steps marked as "Coupled" likely occur simultaneously. (3) The order of some steps, for example substrate loading and ATP loading, may be exchangeable. (4) Certain steps of low $|\Delta G|$ may occur free of others (e.g., ADP dissociation) and are thus absorbed into other steps

P- and A-domains is analogous to the relationship of $\Delta G_{\mathrm{D}}$ to the substrate-transporter affinity: converting from a high-affinity state to a low-affinity state requires energy input. Once the P-domain becomes re-phosphorylated in the next functional cycle, the energy term $-\Delta G_{\mathrm{P}-\mathrm{A}}$ will be released and drive the re-association between $\mathrm{P}$ - and A-domains. The exergonic process of $\mathrm{P}-\mathrm{A}$ association provides all of the energy 
required to drive the efflux of the effective substrates and stores a fraction of the energy in a form of $E_{1}-$ to- $E_{2}$ conformational change (termed $\Delta G_{\mathrm{C}}$ ). Once released, the energy term $-\Delta G_{\mathrm{C}}$ will drive the reversal $\mathrm{E}_{2}$-to- $\mathrm{E}_{1}$ conformational change (see Fig. 3A). In addition, starting from the $E_{1}$ P.S state, the process of P-A association is likely to contain a series of steps in which distinct forms of P-A packing may proceed sequentially through an energetic downhill pathway. The increasingly strong binding between $\mathrm{P}$ - and A-domains is sufficient to drive the substrate ions moving against the electrochemical potential. This scheme may also explain why the predicated high-energy $E_{1}$ P.S state is hard to be (in fact, has never been) captured in structural studies.

Importantly, the $\mathrm{P}-\mathrm{A}$ packing is coupled to the conformational change between $\mathrm{N}_{\mathrm{TM}}$ and $\mathrm{C}_{\mathrm{TM}}$, thus changing the conformation of the P-ATPase from $\mathrm{E}_{1} \mathrm{P} \cdot \mathrm{S}$ to $\mathrm{E}_{2} \mathrm{P} \cdot \mathrm{S}$ (associated with $\Delta G_{\mathrm{C}}$ ) and lowering the affinity of the substrate-binding sites $\left(\Delta G_{\mathrm{D}}\right)$. In agreement with this hypothesis, shortening the M1-A linker by mutation results in impaired transport activity in $\mathrm{Ca}^{2+}$-ATPase (Daiho et al. 2003), presumably by preventing formation of a proper $\mathrm{P}-\mathrm{A}$ interface which would require over tilting of the $\mathrm{C}_{\mathrm{TM}}-\mathrm{P}$ rigid body relative to the membrane. Reversely, extending the linker by mutation results in the transport process being stopped before the substrate $\mathrm{Ca}^{2+}$ is released (Daiho et al. 2007). This observation is probably based on the fact that a longer, more flexible linker prevents effective energy coupling between the $\mathrm{P}-\mathrm{A}$ interaction and the reorganization of the M-domain and thus fails to form a proper substratereleasing channel.

The increasingly stronger $\mathrm{P}-\mathrm{A}$ binding along the conformational pathway from the $\mathrm{E}_{1} \mathrm{P} \cdot \mathrm{S}$ to $\mathrm{E}_{2} \mathrm{P} \cdot \mathrm{S}$ state eventually requires even larger amount of energy to break it, and this energy comes from hydrolysis of the aspartyl-phosphate. In particular, the $\mathrm{P}-\mathrm{A}$ packing energy drives the P-ATPase to the transition state $\mathrm{E}_{2}^{*} \mathrm{P}$ at which the energy-releasing step of dephosphorylation occurs. A similar reasoning of "energy storage in dissociation" has been proposed to explain the ATP hydrolysis cycle in linear ATP-driven motors such as the actomyosin system (Zhang and Feng 2016) as well as ABC transporters (Zhang et al. 2016). In the words of P. Boyer who solved the mystery of rotatory ATP synthase, such a mechanism can be called "binding change mechanism" (Boyer 1998). It is worthy to note that, our energy-coupling scheme is mathematically equivalent to a more common view of the role of ATP hydrolysis. In this "conventional" view (Fig. 3B), the phosphorylation step, i.e., transfer of the $\gamma$-phosphate from ATP to the key Asp residue, directly pushes the P-ATPase system into a high-energy state by dissociating the P-A packing. In turn, the energy gained by the P-ATPase in one power stroke is immediately consumed to drive the uphill $\mathrm{E}_{1} \mathrm{P} \cdot \mathrm{S}$-to- $\mathrm{E}_{2} \mathrm{P} \cdot \mathrm{S}$ transition (Sorensen et al. 2004). However, such a hypothesis would predict the existence of a very stable, low-energy "P-A" packing, from the dephosphorylation step all the way to the next phosphorylation event, and this prediction contradicts with currently available structural information. Therefore, the new scheme of "energy storage in dissociation" provides, in our opinion, a clearer physical picture, although a combination of the two schemes in certain P-ATPases cannot be ruled out.

\section{CONCLUSION REMARKS}

P-ATPases are a family of ubiquitous, primary active transporters. Members of this family seem to share similar structural building blocks and common mechanisms of bidirectional communication between the $\sim 45-\AA$ apart substrate-binding site and the aspartylphosphorylation site. Whereas P-ATPases appear less symmetrical and more complex in domain architecture than their ATPase cousins such as ABC transporters, the underlying thermodynamic principles of P-ATPases are similar to the latter, in particular using the dissociation state as a temporary energy-storage capacitor. The energy landscape proposed here based on the PostAlbers cycle and new structural and kinetic information is expected to facilitate understanding the energycoupling mechanisms of members of the P-ATPase family.

\section{Abbreviations \\ LMCA Listeria monocytogenes $\mathrm{Ca}^{2+}$-ATPase \\ P-ATPase Phosphorylation-type ATPase (transporter) \\ SERCA Sarco(endo)plasmic reticulum $\mathrm{Ca}^{2+}$-ATPase}

Acknowledgements The authors thank Dr. Torsten Juelich (Peking University, China) for linguistic assistance during the preparation of this manuscript. This work was supported by the Ministry of Science and Technology (China) (2015CB910104), the Chinese Academy of Sciences (XDB08020301), and National Natural Science Foundation of China (31470745).

\section{Compliance with Ethical Standards}

Conflict of interest Xuejun C. Zhang and Hongwei Zhang declare that they have no conflict of interest.

Animal rights and informed consent This article does not contain any studies with human or animal subjects performed by any of the authors. 
Open Access This article is distributed under the terms of the Creative Commons Attribution 4.0 International License (http:// creativecommons.org/licenses/by/4.0/), which permits unrestricted use, distribution, and reproduction in any medium, provided you give appropriate credit to the original author(s) and the source, provide a link to the Creative Commons license, and indicate if changes were made.

\section{References}

Albers RW (1967) Biochemical aspects of active transport. Annu Rev Biochem 36:727-756

Apell HJ, Benz G, Sauerbrunn D (2011) Proton diet for the sodium pump. Biochemistry 50:409-418

Bal NC, Maurya SK, Sopariwala DH, Sahoo SK, Gupta SC, Shaikh SA, Pant M, Rowland LA, Bombardier E, Goonasekera SA, Tupling AR, Molkentin JD, Periasamy M (2012) Sarcolipin is a newly identified regulator of muscle-based thermogenesis in mammals. Nat Med 18:1575-1579

Boyer PD (1998) Energy, Life, and ATP (Nobel Lecture). Angew Chem Int Ed Engl 37:2296-2307

Bublitz M, Morth JP, Nissen P (2011) P-type ATPases at a glance. J Cell Sci 124:2515-2519

Daiho T, Yamasaki K, Wang G, Danko S, Iizuka H, Suzuki H (2003) Deletions of any single residues in Glu40-Ser48 loop connecting a domain and the first transmembrane helix of sarcoplasmic reticulum $\mathrm{Ca}(2+)$-ATPase result in almost complete inhibition of conformational transition and hydrolysis of phosphoenzyme intermediate. J Biol Chem 278:39197-39204

Daiho T, Yamasaki K, Danko S, Suzuki H (2007) Critical role of Glu40-Ser48 loop linking actuator domain and first transmembrane helix of $\mathrm{Ca}^{2+}$-ATPase in $\mathrm{Ca}^{2+}$ deocclusion and release from ADP-insensitive phosphoenzyme. J Biol Chem 282:34429-34447

Das A, Rui H, Nakamoto R, Roux B (2017) Conformational transitions and alternating-access mechanism in the sarcoplasmic reticulum calcium pump. J Mol Biol 429:647-666

Dyla M, Terry DS, Kjaergaard M, Sorensen TL, Lauwring Andersen J, Andersen JP, Rohde Knudsen C, Altman RB, Nissen P, Blanchard SC (2017) Dynamics of P-type ATPase transport revealed by single-molecule FRET. Nature 551:346-351

Gourdon P, Liu XY, Skjorringe T, Morth JP, Moller LB, Pedersen BP, Nissen P (2011) Crystal structure of a copper-transporting PIB-type ATPase. Nature 475:59-64

Hill TL (1989) Free energy transduction and biochemical cycle kinetics. Springer, New York

Jardetzky 0 (1966) Simple allosteric model for membrane pumps. Nature 211:969-970

Jensen AM, Sorensen TL, Olesen C, Moller JV, Nissen P (2006) Modulatory and catalytic modes of ATP binding by the calcium pump. EMBO J 25:2305-2314

Kuhlbrandt W (2004) Biology, structure and mechanism of P-type ATPases. Nat Rev Mol Cell Biol 5:282-295

Laursen M, Yatime L, Nissen P, Fedosova NU (2013) Crystal structure of the high-affinity $\mathrm{Na}^{+} \mathrm{K}^{+}$-ATPase-ouabain complex with $\mathrm{Mg}^{2+}$ bound in the cation binding site. Proc Natl Acad Sci USA 110:10958-10963

Liu Y, Liu Y, He L, Zhao Y, Zhang X (2018) Single-molecule fluorescence studies on the conformational change of the ABC transporter MsbA. Biophys Rep 4:153-165

Meissner G, Young RC (1980) Proton permeability of sarcoplasmic reticulum vesicles. J Biol Chem 255:6814-6819
Norimatsu Y, Hasegawa K, Shimizu N, Toyoshima C (2017) Protein-phospholipid interplay revealed with crystals of a calcium pump. Nature 545:193-198

Obara K, Miyashita N, Xu C, Toyoshima I, Sugita Y, Inesi G, Toyoshima C (2005) Structural role of countertransport revealed in $\mathrm{Ca}(2+)$ pump crystal structure in the absence of $\mathrm{Ca}(2+)$. Proc Natl Acad Sci USA 102:14489-14496

Palmgren MG, Nissen P (2011) P-type ATPases. Annu Rev Biophys 40:243-266

Post RL, Hegyvary C, Kume S (1972) Activation by adenosine triphosphate in the phosphorylation kinetics of sodium and potassium ion transport adenosine triphosphatase. J Biol Chem 247:6530-6540

Ratheal IM, Virgin GK, Yu H, Roux B, Gatto C, Artigas P (2010) Selectivity of externally facing ion-binding sites in the $\mathrm{Na} / \mathrm{K}$ pump to alkali metals and organic cations. Proc Natl Acad Sci USA 107:18718-18723

Skou JC (1957) The influence of some cations on an adenosine triphosphatase from peripheral nerves. Biochim Biophys Acta 23:394-401

Sorensen TL, Moller JV, Nissen P (2004) Phosphoryl transfer and calcium ion occlusion in the calcium pump. Science 304:1672-1675

Toyoshima C (2008) Structural aspects of ion pumping by $\mathrm{Ca}^{2+}$. ATPase of sarcoplasmic reticulum. Arch Biochem Biophys 476:3-11

Toyoshima C, Cornelius F (2013) New crystal structures of PIItype ATPases: excitement continues. Curr Opin Struct Biol 23:507-514

Toyoshima C, Nakasako M, Nomura H, Ogawa H (2000) Crystal structure of the calcium pump of sarcoplasmic reticulum at $2.6 \AA$ Å resolution. Nature 405:647-655

Toyoshima C, Nomura H, Tsuda T (2004) Lumenal gating mechanism revealed in calcium pump crystal structures with phosphate analogues. Nature 432:361-368

Toyoshima C, Norimatsu Y, Iwasawa S, Tsuda T, Ogawa H (2007) How processing of aspartylphosphate is coupled to lumenal gating of the ion pathway in the calcium pump. Proc Natl Acad Sci USA 104:19831-19836

Wang FY, Xi QY, Huang KB, Tang XM, Chen ZF, Liu YC, Liang H (2017) Crystal structure, cytotoxicity and action mechanism of $\mathrm{Zn}(\mathrm{II}) / \mathrm{Mn}(\mathrm{II})$ complexes with isoquinoline ligands. J Inorg Biochem 169:23-31

Winther AM, Bublitz M, Karlsen JL, Moller JV, Hansen JB, Nissen P, Buch-Pedersen MJ (2013) The sarcolipin-bound calcium pump stabilizes calcium sites exposed to the cytoplasm. Nature 495:265-269

Zhang XC, Feng W (2016) Thermodynamic aspects of ATP hydrolysis of actomyosin complex. Biophys Rep 2:87-94

Zhang XC, Li H (2019) Interplay between the electrostatic membrane potential and conformational changes in membrane proteins. Protein Sci 28:502-512

Zhang XC, Cao C, Zhou Y, Zhao Y (2014) Proton transfer-mediated GPCR activation. Protein Cell 6:12-17

Zhang XC, Zhao Y, Heng J, Jiang D (2015) Energy coupling mechanisms of MFS transporters. Protein Sci 24:1560-1579

Zhang XC, Han L, Zhao Y (2016) Thermodynamics of ABC transporters. Protein Cell 7:17-27

Zhang XC, Liu M, Lu G, Heng J (2018a) Thermodynamic secrets of multidrug resistance: a new take on transport mechanisms of secondary active antiporters. Protein Sci 27:595-613

Zhang XC, Yang H, Liu Z, Sun F (2018b) Thermodynamics of voltage-gated ion channels. Biophys Rep 4:300-319 